\title{
Análise de interferentes endócrinos na água de abastecimento da cidade de Americana, SP
}

\author{
Analysis of endocrine disruptors in water \\ supply of the city of Americana, SP
}

MARLON Felício ForTi Thaís Delarmelina ${ }^{1}$ Aline da Silva Marques ${ }^{1}$ Patrícia UCelli Simioni ${ }^{1}$ ${ }^{1}$ Faculdade de Americana (FAM, Americana/SP-Brasil
RESUMo Os interferentes endócrinos (IEs) são substâncias que possuem a capacidade de causar efeitos indesejáveis no organismo de animais e seres humanos. A presença de compostos dessa categoria em produtos do cotidiano humano pode causar a contaminação do meio ambiente, principalmente de corpos d'água, devido à ineficiência do tratamento de esgoto. A utilização de água contaminada com IEs pode causar aumento de risco para câncer de mama, testículos e próstata, causar infertilidade e alterações de diversas glândulas do sistema endócrino. O presente artigo aborda uma avaliação da presença de interferentes endócrinos em águas subterrâneas e tratadas para abastecimento, coletadas na cidade de Americana, em São Paulo. As análises das amostras coletadas foram realizadas utilizando o método de cromatografia gasosa acoplada à espectrometria de massas. Os resultados obtidos revelam que os níveis de interferentes endócrinos presentes nas amostras analisadas não ultrapassam os valores considerados normais. Embora não seja possível detectar quantidades reduzidas dos interferentes, vale ressaltar que doses baixas desses interferentes podem causar efeitos colaterais na população. Palavras-chave: Interferentes endócrinos; Água; Meio ambiente; ContaminaÇão; Americana.

AbSTRACT Endocrine Disruptors (EDs) are substances that might cause undesirable effects in animals and humans. The presence of these compounds in human products can cause environment contamination, especially in water systems, due to the inefficiency of sewage treatment. EDs contaminated water can cause increased risk for breast, testis and prostate cancer, causing infertility and alterations in glands of the endocrine system. The present article presents an evaluation of the presence of endocrine disrupters in groundwater and treated water supply, collected in Americana, in São Paulo. The analysis was performed by using the gas chromatography method coupled with mass spectrometry. By the results collected, it was possible to conclude that the levels of endocrine disrupters present in the analyzed samples did not exceed the normal values. Although it is not possible to detect small amounts of the disrupters, it is noteworthy that low doses of these disrupters can cause the side effects in the population.

Keywords: Endocrine Disruptors; Water; Environment; ContaMiNATION; AMERICANA. 


\section{INTRODUÇÃo}

O sistema endócrino é constituído por tecidos e glândulas que produzem hormônios, necessários para a homeostase do organismo de todos os animais. Esse sistema é regulado por mecanismos de feedback positivo e negativo. Os hormônios devem ser mantidos em níveis de equilíbrio para que o indivíduo não apresente alterações morfológicas, sexuais ou na função dos órgãos. ${ }^{1}$ Interferentes endócrinos (IEs), mundialmente denominados como endocrine disruptors (ED), são substâncias ou misturas químicas exógenas (dois ou mais produtos químicos, pesticidas, hormônios ou medicamentos) que, de alguma maneira, interferem no funcionamento do sistema endócrino de espécies animais e de seres humanos. ${ }^{2}$

A presença de contaminantes endócrinos na água é reconhecida, mas, apenas recentemente e com o uso de novos métodos de análise, sua importância tem sido comprovada. Em sua maior parte, tais contaminantes são desprezados em corpos d'água devido à ineficiência de estações de tratamento de esgoto. ${ }^{1}$ Entre os interferentes mais comuns em água estão: ibuprofeno, diclofenaco de sódio, androstano, coprostanol, colesterol, estigmasterol, estrona, estradiol, etinilestradiol, progesterona, levonorgestrel, bisfenol A, 4-nonilfenol, dietilftalato, pentaclorofenol, benzo(a)pireno, cafeína e paracetamol. ${ }^{3}$

Esses compostos exercem diferentes efeitos no organismo humano e no de outros animais, portanto, tais efeitos podem ser classificados por meio da habilidade dos interferentes endócrinos de imitar ou antagonizar os efeitos de hormônios endógenos, e interferir na síntese de hormônios ou de seus receptores. Os principais efeitos da exposição humana a interferentes endócrinos são a diminuição da produção de espermatozoides, aumento de risco de câncer de mama, próstata e testículos, endometriose, problemas de fertilidade em homens e mulheres e alterações na função da glândula tireoide. ${ }^{2,4}$

Entre os interferentes mais comuns encontrados nas águas está o ibuprofeno, fármaco praticamente insolúvel em água e solúvel em algumas soluções aquosas e solventes orgânicos. O uso desregulado desse fármaco pode gerar uma série de problemas como gastrites ou ulceras, pequenas reações alérgicas e urticarias. ${ }^{5}$ Já o diclofenaco de sódio, assim como o ibuprofeno, é um composto não esteroidal. Seu uso pode causar reações alérgicas como dificuldade de respiração, secreção nasal, rash cutâneo e inchaço na face. ${ }^{6}$

O paracetamol é um dos anti-inflamatórios não esteroidais mais utilizados na atualidade. Além de efeitos colaterais conhecidos do paracetamol e outros AINEs, seus possíveis efeitos de interferência endócrina têm sido evidenciados, incluindo diminuição significativa na secreção de testosterona após $24 \mathrm{~h}$ de exposição a paracetamol em estudo in vitro utilizando culturas de células testiculares humanas. ${ }^{7}$

O colesterol também é um esteroide precursor dos hormônios sexuais e de função estrutural de membrana plasmática e responsável por distúrbios arteriais. No meio ambiente, tende a ser mais encontrado em sedimentos. O coprostanol e o colestanol são derivados do colesterol excretado durante seu metabolismo. Por esse motivo pode ser utilizado como marcador de contaminação por esgoto doméstico. ${ }^{8} \mathrm{O}$ estig- 
masterol é um esteroide vegetal, similar ao colesterol e de propriedades estrogênicas. ${ }^{8}$

Ainda, o androstano, a progesterona, a estrona, o levonorgestrel, o estradiol e o etinilestradiol são hormônios sexuais que podem ser facilmente encontrados em água, principalmente de uso doméstico. A exposição a esses hormônios pode causar mudanças nas características masculinas e femininas do organismo humano. ${ }^{9}$

Entre os plásticos o bisfenol A pode ser considerado o maior interferente endócrino, pois além de estar presente em produtos que podem contaminar o ambiente, é também diretamente consumido por estar presente em embalagens de comidas e bebidas. Entre seus efeitos considerados mais importantes estão sua ação na glândula tireoide e sua capacidade estrogênica.$^{10}$

O 4-nonilfenol pertence à classe dos surfactantes e está presente em detergentes, tintas, emulsificantes, plásticos e espermicidas, possui ação estrogênica, tóxica e carcinogênica. ${ }^{11} \mathrm{O}$ dietilftalato e o dibutilftalato são ftalatos de baixa massa molecular utilizados em medicamentos, perfumes, esmaltes, xampus e loções, os ftalatos são considerados interferentes endócrinos pois alteram a função da glândula tireoide. ${ }^{12}$

O pentaclorofenol é uma substância tóxica utilizada como pesticida. Entre seus diversos efeitos colaterais estão as alterações endócrinas causadas por sua ação no hipotálamo e tireoide. ${ }^{13}$ Por outro lado, o benzo(a)pireno faz parte de um grupo de compostos contendo dois ou mais anéis aromáticos condensados, chamados hidrocarbonetos policíclicos aromáticos, estes são compostos formados principalmente pela combustão de matéria orgânica, como durante a queima de carvão, escapamentos de veículos, óleos lubrificantes usados em motores e fumaça de cigarro. O benzo(a)pireno é considerado altamente carcinogênico, embriotóxico e teratogênico, e devido à sua proveniência pode facilmente contaminar alimentos, solo e água. ${ }^{14}$

A presença dessas substâncias pode acarretar diferentes e importantes problemas de saúde e a contaminação do ambiente, por esse motivo é necessária a análise de sua presença e a discussão sobre o uso de tais componentes em produtos do cotidiano. Embora trate-se de um problema de saúde pública, existe uma escassez de estudos para avaliar a presença desses contaminantes.

Assim, o presente trabalho objetivou avaliar a presença de contaminantes endócrinos na água utilizada pelos moradores da cidade de Americana, no interior do estado de São Paulo.

\section{MAterial e Métodos}

O material foi coletado na cidade de Americana, em São Paulo, sendo coletado de poço artesiano e de água de abastecimento no mês de maio de 2018. Após coleta e armazenamento, o preparo das amostras líquidas para a determinação de interferentes endócrinos foi realizado por extração líquido-líquido, como abaixo descrito. Foram realizadas as etapas de extração, identificação e quantificação dos compostos. A metodologia de análise foi realizada no laboratório Mérieux Nutrisciences, de Piracicaba (SP).

\section{1. Amostras}

Foram coletados dois tipos de amostras, água tratada e água de poço artesiano. Após 
limpeza das mãos e com uso de luvas, a água da torneira foi coletada, após ser desprezada a vazão inicial, ou seja, após 2 minutos. Realizou-se a análise dos parâmetros de campo como $\mathrm{pH}$, temperatura da água, e quantidade de cloro. Utilizou-se frasco de vidro âmbar para armazenar a amostra, logo depois da coleta, preservaram-se as amostras em caixa de isopor com gelo, mantendo a temperatura entre 0 e 6 graus até o momento da extração, sendo que nessas condições de refrigeração a amostra pode ser armazenada por até 14 dias. Os procedimentos foram registrados em ficha de coleta, a qual acompanha a amostra para consequente registro e etiquetagem no laboratório. A amostra foi armazenada em geladeira até seu uso.

\section{2. Solventes, reagentes e padrões}

Foram utilizados diclorometano, ácido sulfúrico, sulfato de sódio anidro, hidróxido de sódio $10 \mathrm{M}$, padrões de referência dos compostos descritos, devidamente certificados, água ultrapura e água deionizada. Os padrões dos interferentes (puros ou em solução), utilizados como materiais de referência certificados foram: ibuprofeno, diclofenaco de sódio, androstano, coprostanol, colestanol, colesterol, estigmasterol, estrona, estradiol, progesterona, bisfenol A, 4-nonilfenol, pentaclorofenol, benzo(a)pireno, cafeína e paracetamol (Absolute Standards, Inc).

\section{3. Procedimentos}

\section{3. 1. Extração dos compostos}

A amostra previamente mantida a $4^{\circ} \mathrm{C}$, foi estabilizada à temperatura ambiente. Em um funil de separação de 2,0L, foi adi- cionado 1,0L da amostra. $\mathrm{O}$ pH foi aferido, com registro dos valores obtidos. Valores diferentes de $\mathrm{pH}$ 7,0 foram corrigidos com soluções de $\mathrm{NaOH}$ ou $\mathrm{H}_{2} \mathrm{SO}_{4}$. Em seguida, adicionou-se $400 \mu \mathrm{L}$ do padrão 8270 (base/ neutral \& acid surrogate standard, concentração de $4000 \mathrm{mg} / \mathrm{L}$, (Absolute Standards) em $1,0 \mathrm{~mL}$ de amostra. Adicionou-se $60 \mathrm{~mL}$ de diclorometano e agitou-se vigorosamente por no mínimo 1 minuto, tomando-se o cuidado de abrir periodicamente o funil para a saída dos gases formados. Transferiu-se a fase orgânica para um balão de fundo chato, passando por um funil de transferência contendo papel de filtração rápida e sulfato de sódio. Repetiram-se as fases de adição de diclorometano e transferência de fase orgânica. Novamente o $\mathrm{pH}$ foi corrigido para o valor inferior a 2,0 com ácido sulfúrico. Repetiu-se as fases de adição de diclorometano e transferência de fase orgânica mais duas vezes, a fase aquosa foi descartada e a fase orgânica recolhida no balão de fundo chato.

\section{3. 2. Concentração das amostras}

A fase orgânica foi concentrada em volume aproximado de $1 \mathrm{mLem}$ rota-evaporador com banho maria à $45^{\circ} \mathrm{C}$ (CBLCB ou no concentrador automático [BUCHI Brasil Ltda). Em seguida, transferiu-se o extrato para um tubo de $2 \mathrm{~mL}$, com auxílio de uma pipeta Pasteur, lavou-se muito bem o balão com diclorometano e transferiu-se novamente para o tubo de $2 \mathrm{~mL}$. Novamente concentrou-se a fase orgânica até $1 \mathrm{~mL}$ com fluxo baixo de gás inerte (TecVap LV, TurboVap ${ }^{\circledR}$ LV - Biotage), cuidadosamente para evitar a perda de compostos.

Os extratos foram armazenados em tubos de $2 \mathrm{~mL}$, devidamente identificados e 
conservados refrigerados até o momento da análise. O controle negativo, contendo apenas água deionizada, sofreu os mesmos procedimentos de extração e injeção das amostras analisadas, para a verificação de possíveis contaminações durante todo o processo. Os controles internos do equipamento foram utilizados para controlar e monitorar o desempenho do preparo, manuseio de itens de ensaio, condições do equipamento (calibração/operação), e o processo analítico global (extração/concentração/equipamento/análise).

Esses foram adicionados a uma concentração de $10 \mathrm{mg} / \mathrm{L}$ para as amostras líquidas extraídas e suas recuperações foram dentro da faixa limite (40-140\%). Para o preparo dos padrões, em vidraria calibrada de $10 \mathrm{~mL}$, foi diluído $62,5 \mu \mathrm{L}$ do padrão 8270 (base/neutral \& acid surrogate standard, concentração de $4000 \mathrm{mg} / \mathrm{L}$ ), fabricado pela marca Absolute Standards, Inc. em volume de diclorometano suficiente para um volume final de $10 \mathrm{~mL}$ de solução. Essa solução de trabalho estava em uma concentração de $25 \mathrm{mg} / \mathrm{L}$. O LCS é uma amostra de controle com os compostos alvos da análise em concentração conhecida. Ela foi utilizada para verificar a eficiência global do processo de análise. Uma amostra de LCS deve ser feita a cada grupo de no máximo 20 amostras. A extração foi realizada utilizando $1,0 \mathrm{~L}$ de água deionizada, adicionando-se $100 \mu \mathrm{L}$ da mistura de interferentes $(100 \mathrm{mg} / \mathrm{L})$.

\section{3. 3. Método de cromatografia gasosa acoplada à espectrometria de massas para análise de interferentes}

A determinação dos interferentes endócrinos foi realizada por cromatografia gasosa acoplada à espectrometria de massas (GC/ MS) em amostras liquidas de água tratada para consumo humano, extraídas com solvente orgânico (partição líquido-líquido), identificados e quantificados por GC/MS. Também conhecida como partição, a extração líquido-líquido é um método utilizado para separar compostos baseado em suas diferentes solubilidades em dois líquidos diferentes e imiscíveis, normalmente água e um solvente orgânico. A eficiência da extração depende da afinidade do soluto pelo solvente de extração, da razão das fases e dos números de extrações, de acordo com a metodologia definida pelo Laboratório Mérieux Nutrisciences, de Piracicaba, São Paulo.

Os analitos eluídos na coluna capilar foram introduzidos no espectrômetro de massa por meio da interface. A identificação de analitos alvo foi realizada comparando seus espectros de massas com os espectros de padrões autênticos. A quantificação foi realizada comparando a resposta de um íon principal relativo usando uma curva de calibração. Para o desenvolvimento dos métodos foram utilizados o cromatógrafo gasoso modelo 7890, acoplado ao espectrômetro de massas modelo 5975, acoplado com coluna com a fase $5 \%$ fenilmetilsiloxano - $20 \mathrm{~m}$; ID $180 \mu \mathrm{m}$; film $0,18 \mu \mathrm{m}$, todos os equipamentos foram produzidos pela marca Agilent.

\section{Análises}

Todos os cálculos dos resultados foram realizados pelo Software de gerenciamento de informações myLIMS. Os dados foram obtidos por meio de software para integração e operação do equipamento, Data Analysis, fornecido pelo fabricante do equi- 
pamento e software para gerenciamento das informações, myLIMS, desenvolvido pelo laboratório de análise. Foi realizada uma réplica de cada amostra extraída, ou seja, uma duplicata para a amostra de água tratada e uma para a água subterrânea. As análises de dados não se aplicam, visto que todos os resultados obtidos estavam abaixo dos valores de referência.

\section{RESUltados}

Os resultados obtidos nas análises de interferentes endócrinos nas amostras de água tratada e água subterrânea da cidade de Americana (SP), estão apresentados na tabela 1. Os parâmetros analisados mostram resultados abaixo da faixa de referência considerada mensurável para os analitos e pelo método aqui apresentados, indicando que a presença de interferentes endócrinos nas amostras analisadas não ultrapassa a quantidade considerada limite. Assim, pela análise, pode-se observar que não foi registrada a presença de interferentes endócrinos nas amostras de água obtidas na cidade de Americana (SP), tratadas ou não, coletadas no período de $21 \mathrm{~h}$ horas, do dia 05/05/2017.

TABELA 1 - Resultados das análises realizadas na água subterrânea e na água tratada.

\begin{tabular}{|c|c|c|c|c|}
\hline Parâmetros & Unidade & $\begin{array}{c}\text { Limite } \\
\text { Quantitativo/ } \\
\text { Faixa }\end{array}$ & Água subterrânea & Água tratada \\
\hline 5-Alfa androstano & $\mu \mathrm{g} / \mathrm{L}$ & 1 & $<1$ & $<1$ \\
\hline $17 \alpha$-etinilestradiol & $\mu \mathrm{g} / \mathrm{L}$ & 5 & $<5$ & $<5$ \\
\hline 4-nonilfenol & $\mu \mathrm{g} / \mathrm{L}$ & 1 & $<1$ & $<1$ \\
\hline Benzo(a)pireno & $\mu \mathrm{g} / \mathrm{L}$ & 1 & $<1$ & $<1$ \\
\hline Bisfenol A & $\mu \mathrm{g} / \mathrm{L}$ & 1 & $<1$ & $<1$ \\
\hline Colesterol & $\mu \mathrm{g} / \mathrm{L}$ & 1 & $<1$ & $<1$ \\
\hline Coprostanol & $\mu \mathrm{g} / \mathrm{L}$ & 1 & $<1$ & $<1$ \\
\hline Norgestrel & $\mu \mathrm{g} / \mathrm{L}$ & 5 & $<5$ & $<5$ \\
\hline Diclofenaco de Sódio & $\mu \mathrm{g} / \mathrm{L}$ & 1 & $<1$ & $<1$ \\
\hline Estradiol & $\mu \mathrm{g} / \mathrm{L}$ & 5 & $<5$ & $<5$ \\
\hline Estrona & $\mu \mathrm{g} / \mathrm{L}$ & 1 & $<1$ & $<1$ \\
\hline Ibuprofeno & $\mu \mathrm{g} / \mathrm{L}$ & 1 & $<1$ & $<1$ \\
\hline Pentaclorofenol & $\mu \mathrm{g} / \mathrm{L}$ & 1 & $<1$ & $<1$ \\
\hline Progesterona & $\mu \mathrm{g} / \mathrm{L}$ & 5 & $<5$ & $<5$ \\
\hline Estigmasterol & $\mu \mathrm{g} / \mathrm{L}$ & 5 & $<5$ & $<5$ \\
\hline Dibutilftalato & $\mu \mathrm{g} / \mathrm{L}$ & 1 & $<1$ & $<1$ \\
\hline Dietilftalato & $\mu \mathrm{g} / \mathrm{L}$ & 1 & $<1$ & $<1$ \\
\hline Colestanol & $\mu \mathrm{g} / \mathrm{L}$ & 1 & $<1$ & $<1$ \\
\hline Cafeína & $\mu \mathrm{g} / \mathrm{L}$ & 1 & $<1$ & $<1$ \\
\hline Paracetamol & $\mu \mathrm{g} / \mathrm{L}$ & 1 & $<1$ & $<1$ \\
\hline
\end{tabular}




\section{DisCUSSÃo}

Atualmente, existe um grande interesse de setores públicos e privados em discutir os problemas gerados para o meio ambiente, com a criação de normas e condutas, estabelecendo, assim, padrões para a diminuição de resíduos e compostos químicos com grande potencial em poluição. ${ }^{15}$

Desde 1996 o Departamento de Pesquisas e Desenvolvimento da Agência de Proteção Ambiental dos Estados Unidos (USEPA), vem comprovando, por meio de estudos científicos, a importância ambiental da identificação de interferentes endócrinos, com destaque para produtos químicos da indústria química e farmacêutica, os quais causam efeitos adversos ao meio ambiente aquático e à saúde pública. ${ }^{1}$

As substâncias classificadas como interferentes endócrinos têm a capacidade de se ligar aos receptores que são utilizados pelos hormônios e promover alterações na síntese, secreção, metabolismo e/ou ação hormonais, sendo assim, são capazes de interferir nas funções biológicas. ${ }^{16,17}$

De forma similar ao presente trabalho, Silva e colegas avaliaram a presença de interferentes endócrinos por ensaios in vivo, em diferentes áreas da região metropolitana da cidade de São Paulo (SP) e observaram presença de proteína vitelogenina no plasma de peixes. ${ }^{17}$ Já segundo Calafat e colegas, das 371 amostras de urina de americanos adultos analisadas foram encontrados bisfenol A em cerca de $95 \%$ e nonilfenol em cerca de $51 \% .^{18}$

Utilizando-se de testículos de fetos abortados e aparentemente normais, Lambrot e colaboradores avaliaram os efeitos do ftalato em tecidos humanos. Eles observaram que em uma certa concentração de ftalato, houve uma diminuição da expressão de RNAm de hormônio antimülleriano e do número de células germinativas. Esse foi o primeiro experimento que demonstrou que ftalatos modificam o desenvolvimento da linhagem de células germinativas e que apresentam efeito prejudicial potencialmente forte em relação à fertilidade masculina na vida adulta. ${ }^{19}$

Em uma pesquisa realizada em águas superficiais na região de Campinas (SP), foram investigadas amostras de água coletadas em seis pontos localizados na bacia do rio Atibaia. Dentre os compostos avaliados, foi encontrado níveis aumentados de $17 \beta$-estradiol, o $17 \alpha$-etinilestradiol paracetamol (acetaminofeno), o ácido acetilsalicílico, a cafeína, o di-n-butil ftalato em mais de $66 \%$ das amostras e ao menos uma vez foi detectado bisfenol A. Ainda, foram observados níveis elevados de cafeína, bisfenol A, $17 \beta$-estradiol, o $17 \alpha$-etinilestradiol em um ponto a jusante da cidade. ${ }^{20}$ Swan e colaboradores comprovaram que hormônios usados para o crescimento do gado, assim como pesticidas usados para melhoria da pastagem, foram responsáveis pela redução da taxa de espermatozoides em homens cujas mães ingeriram muita carne vermelha durante sua gestação. ${ }^{20}$

A Companhia Ambiental do Estado de São Paulo (CETESB), apresenta diretrizes para a análise de interferentes na água de abastecimento, em específico para as dosagens de interferentes. Nesse contexto, o presente resultado mostra valores considerados como permitidos. ${ }^{21,22}$ Entretanto, cabe ressaltar que, embora a cromatografia gasosa 
acoplada à espectrometria de massas (GC/ MS) seja considerada o padrão ouro para a análise de interferentes na água, entre as limitações do presente estudo está a coleta única da amostra, a qual pode afetar o resultado obtido e limitar a análise do resultado ao período de coleta.

\section{ConClusão}

Os resultados obtidos a partir da análise de amostras de água tratada e subterrânea da cidade de Americana (SP), indicam que as quantidades encontradas de interferentes endócrinos estão dentro do limite permitido e abaixo do limite de detecção do método utilizado. Os resultados aqui obtidos permitem concluir que a fonte de água da cidade de Americana (SP) avaliada não apresenta resultados positivos nas amostras coletadas no mês de maio de 2017. Isso permite concluir que, na data de coleta realizada, essas fontes não apresentavam risco associados às substâncias aqui analisadas (5-alfa androstano, 17a-etinilestradiol, 4-nonilfenol, benzo(a) pireno, bisfenol A, colesterol, coprostanol, norgestrel, diclofenaco de sódio, estradiol, estrona, ibuprofeno, pentaclorofenol, progesterona, estigmasterol, dibutilftalato, dietilftalato, colestanol, cafeína e paracetamol).

Embora o presente método não permita mensurar quantidades reduzidas dos interferentes, vale ressaltar que a presença dos interferentes endócrinos na água, mesmo em baixas quantidades, pode causar os efeitos colaterais na população da cidade exposta à essas substâncias presentes no meio ambiente.

Assim, sugere-se a realização de outros estudos para que permitam conclusões mais aprofundadas, bem como que estudos futuros trabalhem com períodos diferentes de coleta, para estimar melhor a quantidade de tais interferentes.

\section{REFERÊNCIAS}

1. Simões AP, Silva M, Mange N, Feitosa VH, Cardoso T, Quináglia GA. A presença de interferentes endócrinos em águas superficiais e de abastecimento: um problema da vida moderna? Rev. Eletrônica Gest., Educ. Tecnol. Ambient.2014; 54:6-16.

2. Ghiselli G, Jardim WF. Interferentes endócrinos no ambiente. Qim. nova.2007; 30(3):695-706

3. Snyder SA, Westerhoff P, Yoon Y, Sedlak DL. Pharmaceuticals, personal care products, and endocrine disruptors in water: implications for the Water industry. 2003; 20(5):449-70.

4. Mendes JJA. The endocrine disrupters: a major medical challenge. Food Chem Toxicol. 2002; 40(6):781-8.

5. Marques L. O Ibuprofeno: Um fármaco com sucesso. Cent Quim e Univ Évora. 2009, p. 1-3.

6. Souza RL, Tubino M. Spectrophotometric determination of diclofenac in pharmaceutical preparations.J. Braz. Chem. Soc.2005; 16(5):1068-73.

7. Albert O, Desdoits-Lethimonier C, Lesné L, Legrand A, Guillé F, Bensalah K et al. Paracetamol, aspirin and indomethacin display endocrine disrupting properties in the adult human testis in vitro. Hum Reprod. 2013; 28(7):1890-8.

8. Gishelli G. Avaliação da qualidade das aguas destinadas ao abastecimento publico na região de Campinas: ocorrencia e determinação dos interferentes endocrinos (IE) e produtos farmaceuticos e de higiene pessoal (PFHP) [Tese]. Campinas, SP: Universidade Estadual de Campinas, Instituto de Quimica; 2001.211 p. 
9. Otomo, JI. Contribuição antrópica na qualidade das águas da represa do Guarapiranga. Um estudo sobre interferentes endócrinos [Tese]. São Paulo: Instituto de Pesquisas Energéticas e Nucleares; 2015

10. Costa EMF, Spritzer PM, Hohl A, Bachega TASS. Effects of endocrine disruptors in the development of the female reproductive tract. Arq Bras Endocrinol Metabol. 2014; 58(2):153-61

11. Spadoto M. Análise dos efeitos tóxicos do nonilfenol e do bisfenol A em organismos de água doce [Dissertação]. São Carlos: Universidade de São Paulo, Escola de Engenharia de São Carlos; 2013

12. Silva MN. Determinação de desreguladores endocrinos na água e no sedimentos do açude Santo Anastácio na cidade de Fortaleza/Ce [Dissertação]. Fortaleza: Universidade Federal do Ceará; 2016

13. Souza RF. Investigação da presença de resíduo de pentaclorofenol em gelatina comestível por cromatografia a gás com captura de elétrons (CG/DCE)[Dissertação]. Porto Alegre: Universidade Federal do Rio Grande do Sul; 2008. 75 p.

14. Caruso MSF, Alaburda J. Hidrocarbonetos policíclicos aromáticos -benzo(a)pireno: uma revisão. Rev. Inst. Adolfo Lutz. 2008; 67:1-27.

15. Sodré FF, Locatelli MAF, Montagner CC, Jardim WF. Origem e destino de Interferentes endócrinos em águas naturais. Cad temático. 2007; 6: 1-27

16. Fontenele EGP, Martins MRA, Quidute ARP, Montenegro RM Júnior. Contaminantes ambientais e os interferentes endócrinos. Arq Bras Endocrinol Metab. 2010; 54 (1): 6-16

17. Silva AL. Interferentes endócrinos no meio ambiente: um estudo de caso em amostras de água in natura e efluente de estação de tratamento de esgotos da região metropolitana de São Paulo [tese]. São Paulo: , Faculdade de Saúde Pública; 2009

18. Calafat AM, Kuklenyik Z, Reidy JA, Caudill SP, Ekong J, Neddham LL. Urinary Concentrations of Bisphenol A and 4-Nonylphenol in a Human Reference Population. Environ Health Perspect. 2005; 113(4):391-5.

19. Lambrot R, Muczynski V, Lécureuil C, Angenard G, Coffigny H, Pairault C, et al. Phthalates Impair Germ Cell Development in the Human Fetal Testis in Vitro. Environ Health Perspect. 2009; 117(1):32-7.

20. Sodré FF, Montagner CC, Locatelli MAF, Jardim WF. Ocorrência de Interferentes Endócrinos e Produtos Farmacêuticos em Águas Superficiais da Região de Campinas (SP, Brasil). J. Braz. Soc. Ecotoxicol. 2007; 2(2):187-96.

21. CETESB (BR). Companhia, de Tecnologia de Saneamento Ambiental. Qualidade das Águas Doces no Estado de São Paulo | Apêndice E - Significado Ambiental e Sanitáriodas Variáveis. 2009.

22. Reis FO, Martinez EA, Silva FMR, Lima ACR. Análise dos dispositivos legais nos estados brasileiros perante a Resolução 420/2009 [Internet]. Rio Grande; 2017. Disponível em: https://ambitojuridico.com.br/cadernos/direito-ambiental/analise-dos-dispositivos-legais-nos-estados-brasileiros-perante-a-resolucao-420-2009/.

\section{DAdos dos AUTORES}

\section{Aline da Silva Marques}

Bacharel em biomedicina pela Faculdade de Americana (FAM), Americana/SP - Brasil. aline-marques2002@hotmail.com 


\section{Thaís Delarmelina}

Bacharel em biomedicina pela Faculdade de Americana (FAM), Americana/SP - Brasil. tdelarmelina@gmail.com

\section{MARLON Felício Forti}

Coordenador de laboratório Merieux Nutrisciences.marlon.forti@mxns.com

\section{Patrícia Ucelli Simioni}

Coordenadora do curso de Biomedicina e Tecnologia em Estética e Cosmética e Técnicos em SaúdeFaculdade de Americana (FAM), Americana/SP - Brasil. Docente de do curso de Medicina - Universidade Anhembi Morumbi- Piracicaba/SP - Brasil. psimioni@gmail.com

Submetido em: 18-10-2018

Aceito em: 10-5-2021 